\title{
ANTITUBERCULAR ACTIVITY OF SOME NEWER 6-PYRIDAZINONE DERIVATIVES
}

\author{
Asif Husain $^{\mathrm{a} *}$, Aftab Ahmad ${ }^{\mathrm{b}}$, Anil Bhandari ${ }^{\mathrm{b}}$ and Veerma Ram ${ }^{\mathrm{b}}$

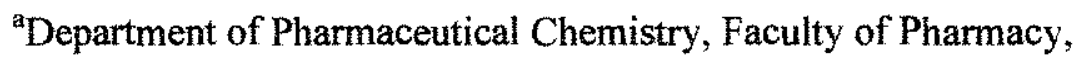 \\ Hamdard University, New Delhi-110062, India, \\ Email:drasifhusain@yahoo.com,ahusain@jamiahamdard.ac.in \\ ${ }^{b}$ Faculty of Pharmaceutical Sciences, \\ Jodhpur National University, Jodhpur, Rajasthan-342001, India
}

\begin{abstract}
Two series of 6-pyridazinone derivatives (17-30) were synthesized and evaluated for antitubercular activities against Mycobacterium tuberculosis $H_{37} R v$ strain. The results indicated that among the synthesized compounds, 5-(4-hydroxy-3-methoxybenzyl)-3-phenyl-1,6-dihydro-6-pyridazinone (23) showed good antitubercular activity. Three more compounds, $(18,25 \& 27)$ were significant in their antitubercular action. The present study reveals the antitubercular potential of 6-pyridazinones.
\end{abstract}

KEY WORDS: Pyridazinone, antitubercular, mycobacteria, furanone.

\section{RESUMO}

Duas séries de derivados de 6-piridazona foram sintetizados e avaliados para a atividade antitubercular contra Mycobacterium tuberculosis da cepa $H_{3} R v$. Os resultados experimentais indicaram que o composto 5-(4-hidroxi-3-metoxibenzil)-3-fenil-1,6-dihidro-6-piridazinona (23) apresentou boa atividade antitubercular. Outros três compostos $(18,25$ e 27) mostraram atividade antitubercular significativa. O presente estudo revela o potencial antitubercular de 6-piridazinonas.

PALAVRAS-CHAVE: Piridazinona, atividade contra tuberculose, micobactéria, furanona.

VISIT OUR SITE: http:/www.sbjchem.he.com.br 


\section{INTRODUCTION}

Resistance of Mycobacterium tuberculosis strains to available antitubercular drugs is an increasing problem worldwide. New potent antimycobacterial drugs with new mechanisms of action have not been developed in the last forty years ${ }^{1}$.TB is considered by the WHO to be the most important chronic communicable disease in the world. About $32 \%$ of the world's population is currently infected with TB. The emergence of AIDS, decline of socioeconomic standards and a reduced emphasis on tuberculosis control programs contribute to the disease's resurgence in industrialized countries ${ }^{2}$. If the present trend continues, tuberculosis is likely to claim more than 30 million lives within the new decade. A great deal of research is being directed towards the development of new antitubercular drug.

During recent years pyridazinones have been a subject of intensive research due to their wide spectrum of biological activities. Substituted pyridazinones have been found to have potent antibacterial, antifungal and antiviral including anti-HIV activities ${ }^{3-6}$. Various $3-(2 H)-$ pyridazinone derivatives have shown anticancer ${ }^{6}$, analgesic $\&$ anti-inflammatory ${ }^{6-8}$, anticonvulsant ${ }^{9}$, cardiotonic \& hypotensive $e^{10,11}$ and antiulcer activities ${ }^{12}$. Now research efforts are toward the search of new antimycobacterial agents (new classes of compounds), which are structurally different from known antimycobacterial drugs ${ }^{13,14}$. The present work describes the synthesis of newer $2(3 \mathrm{H})$-pyridazinones with encouraging antitubercular activity.

\section{MATERIALS AND METHODS}

\section{Synthesis}

Melting points were determined in open capillary tubes and are uncorrected. Thin-layer chromatography was carried out to monitor the reactions using silica gel $G$ plates. The IR spectra were recorded in potassium bromide pellets using a Perkin-Elmer $1725 \mathrm{X}$ spectrophotometer. Elemental analyses were performed on a Perkin-Elmer 240 analyzer and the values were in range of $\pm 0.4 \%$ theoretical value for the element analyzed $(\mathrm{C}, \mathrm{H}, \mathrm{N}){ }^{1} \mathrm{H}-\mathrm{NMR}$ spectra were recorded on Bruker spectropsin DPX-300 MHz in $\mathrm{CDCl}_{3}$; chemical shift $(\delta)$ values are reported in parts per million (ppm). The splitting pattern abbreviations are as follows: $s$, singlet; $d$, doublet; $m$, multiplet. Mass spectra under electron impact conditions (EI) were recorded at $70 \mathrm{eV}$ ionizing voltage with a VG Prospec instrument and are presented as $\mathrm{m} / \mathrm{z}$. Spectral data are consistent with the assigned structures.

Preparation of 3-(4-Chloro/methyl benozyl)propionic acid (1,2). The compounds, 1 and 2, were synthesized according to the reported method ${ }^{14}$.

Preparation of 3-Arylidene-5-(4-chloro/methyl phenyl)-2(3H)-furanones (3-16). Compounds (316) were synthesized from 3-(4-chloro/methyl benozyl)propionic acid $(1,2)$ following literature method $^{14}$.

General Procedure for the synthesis of 5-(substituted benzyl)-3-aryl-1,6-dihydro-6-pyridazinones $(17-30)$. 2(3H)-Furanones (3-16) $(0.005 \mathrm{~mole})$ and hydrazine hydrate $(1-2 \mathrm{~mL})$ in $n$-propanol (5$6 \mathrm{~mL}$ ) were refluxed for $3 \mathrm{~h}$. After refluxing reaction mixture was poured onto crushed ice, a precipitate was obtained, which was filtered, dried and recrystallized from methanol to give TLC pure 5-(substituted benzyl)-3-aryl-1,6-dihydro-6-pyridazinone derivatives (17-30). 


\section{SOUTH. BRAZ. J. CHEM., Vol.19, No. 19, 2011}

\section{A. Husain, A. Ahmad, A. Bhandari and V. Ram}

5-Benzyl-3-phenyl-1,6-dihydro-6-pyridazinone (17): Yield: $58 \%$; m.p. $168-170{ }^{\circ} \mathrm{C}$; ${ }^{\mathrm{H}} \mathrm{H}-\mathrm{NMR}$ $\left(\mathrm{CDCl}_{3}, \delta, \mathrm{ppm}\right): 3.99\left(\mathrm{~s}, 2 \mathrm{H}, \mathrm{CH}_{2}\right), 7.26(\mathrm{~s}, 1 \mathrm{H}, \mathrm{H}-4$, pyridazinone ring $), 7.29-7.41(\mathrm{~m}, 6 \mathrm{H}$, $2 \times \mathrm{H}-2,4,6$, benzyl + phenyl), 7.58-7.65 (m, 4H, 2xH-3,5, benzyl + phenyl), 10.62 (s, $1 \mathrm{H}, \mathrm{NH})$; MS $(\mathrm{m} / \mathrm{z}): 262\left(\mathrm{M}^{+}\right) ;$IR $\left(\mathrm{cm}^{-1}, \mathrm{KBr}\right): 3186(\mathrm{NH}), 2949(\mathrm{CH}), 1683(\mathrm{CO}) ;$ Anal calcd. for $\mathrm{C}_{17} \mathrm{H}_{14} \mathrm{~N}_{2} \mathrm{O}(\mathrm{CHN})$

5-(2-Chlorobenzyl)-3-phenyl-1,6-dihydro-6-pyridazinone (18): Yield: $63 \%$; m.p. $210{ }^{\circ} \mathrm{C} ;{ }^{1} \mathrm{H}-$ NMR $\left(\mathrm{CDCl}_{3}, \delta, \mathrm{ppm}\right): 4.42\left(\mathrm{~s}, 2 \mathrm{H}, \mathrm{CH}_{2}\right), 7.24(\mathrm{~s}, 1 \mathrm{H}, \mathrm{H}-4$, pyridazinone ring), 7.26-7.64 (m, $9 \mathrm{H}$, phenyl+ benzyl) $10.77(\mathrm{~s}, 1 \mathrm{H}, \mathrm{NH}) ; \mathrm{MS}(\mathrm{m} / \mathrm{z}): 296 / 297\left(\mathrm{M}^{+} / \mathrm{M}+1\right) ; \mathrm{IR}\left(\mathrm{cm}^{-1}, \mathrm{KBr}\right): 3179$ $(\mathrm{NH}), 2942(\mathrm{CH}), 1688(\mathrm{CO}), 726(\mathrm{C}-\mathrm{Cl})$; Anal calcd. for $\mathrm{C}_{17} \mathrm{H}_{13} \mathrm{ClN}_{2} \mathrm{O}(\mathrm{CHN})$.

5-(4-Chlorobenzyl)-3-phenyl-1,6-dihydro-6-pyridazinone (19): Yield: $68 \%$; m.p. $188{ }^{\circ} \mathrm{C} ;{ }^{1} \mathrm{H}$ $\mathrm{NMR}\left(\mathrm{CDCl}_{3}, \delta, \mathrm{ppm}\right): 3.93\left(\mathrm{~s}, 2 \mathrm{H}, \mathrm{CH}_{2}\right), 7.24$ (s, $1 \mathrm{H}, \mathrm{H}-4$, pyridazinone ring), $7.27 \& 7.67$ (d, each, $2 \mathrm{xA}_{2} \mathrm{~B}_{2}, p$-chlorophenyl), $7.30(\mathrm{~m}, 1 \mathrm{H}, \mathrm{H}-4$, phenyl), $7.33(\mathrm{~m}, 2 \mathrm{H}, \mathrm{H}-2,6$, phenyl), 7.46 (m, $2 \mathrm{H}, \mathrm{H}-3,5$, phenyl), $12.68(\mathrm{~s}, 1 \mathrm{H}, \mathrm{NH}) ; \mathrm{MS}(\mathrm{m} / \mathrm{z}): 296 / 297\left(\mathrm{M}^{+} / \mathrm{M}+1\right) ; \mathrm{IR}\left(\mathrm{cm}^{-1}, \mathrm{KBr}\right): 3173$ $(\mathrm{NH}), 2936(\mathrm{CH}), 1672(\mathrm{CO}), 707(\mathrm{C}-\mathrm{Cl})$; Anal calcd. for $\mathrm{C}_{17} \mathrm{H}_{13} \mathrm{ClN}_{2} \mathrm{O}(\mathrm{CHN})$.

5-(3-Nitrobenzyl)-3-phenyl-1,6-dihydro-6-pyridazinone (20): Yield: $58 \%$, m.p. $178-180{ }^{\circ} \mathrm{C}$; ${ }^{1} \mathrm{H}-$ NMR $\left(\mathrm{CDCl}_{3}, \delta, \mathrm{ppm}\right): 3.89\left(\mathrm{~s}, 2 \mathrm{H}, \mathrm{CH}_{2}\right), 7.14(\mathrm{~s}, 1 \mathrm{H}, \mathrm{H}-4$, pyridazinone ring $), 7.26(\mathrm{~m}, 1 \mathrm{H}, \mathrm{H}-$ 4, phenyl), 7.31 (m, 1H, H-4, phenyl), $7.44(\mathrm{~m}, 1 \mathrm{H}, \mathrm{H}-6$, benzyl ring), $7.34(\mathrm{~m}, 2 \mathrm{H}, \mathrm{H}-2,6$, phenyl), $7.50(\mathrm{~m}, 2 \mathrm{H}, \mathrm{H}-3,5$, phenyl), $8.14(\mathrm{~m}, 1 \mathrm{H}, \mathrm{H}-5$, benzyl ring $), 8.16(\mathrm{~m}, 1 \mathrm{H}, \mathrm{H}-4$, benzyl ring), $8.18\left(\mathrm{~m}, 1 \mathrm{H}, \mathrm{H}-2\right.$, benzyl ring), $10.98(\mathrm{~s}, 1 \mathrm{H}, \mathrm{NH}) ; \mathrm{MS}(\mathrm{m} / \mathrm{z}): 307\left(\mathrm{M}^{+}\right) ; \mathrm{IR}\left(\mathrm{cm}^{-1}, \mathrm{KBr}\right)$ : $3178(\mathrm{NH}), 2942(\mathrm{CH}), 1686(\mathrm{CO})$; Anal calcd. for $\mathrm{C}_{17} \mathrm{H}_{13} \mathrm{~N}_{3} \mathrm{O}_{3}(\mathrm{CHN})$.

5-(4-Methoxybenzyl)-3-phenyl-1,6-dihydro-6-pyridazinone (21): Yield: $52 \%$; m.p. $186{ }^{\circ} \mathrm{C} ;{ }^{1} \mathrm{H}$ $\mathrm{NMR}\left(\mathrm{CDCl}_{3}, \delta, \mathrm{ppm}\right): 3.82\left(\mathrm{~s}, 3 \mathrm{H}, \mathrm{OCH}_{3}\right), 3.93\left(\mathrm{~s}, 2 \mathrm{H}, \mathrm{CH}_{2}\right), 6.82(\mathrm{~s}, 1 \mathrm{H}, \mathrm{H}-4$, pyridazinone ring), $6.83 \& 7.41$ (d, each, $2 \mathrm{xA}_{2} \mathrm{~B}_{2}, p$-methoxy benzyl ring), $7.21(\mathrm{~m}, 1 \mathrm{H}, \mathrm{H}-4$, phenyl ring), 7.27 (m, 2H, H-2,6, phenyl ring), $7.65(\mathrm{~m}, 2 \mathrm{H}, \mathrm{H}-3,5$, phenyl ring), $10.87(\mathrm{~s}, 1 \mathrm{H}, \mathrm{NH}) ; \mathrm{MS}(\mathrm{m} / \mathrm{z})$ : $292\left(\mathrm{M}^{+}\right)$; IR $\left(\mathrm{cm}^{-1}, \mathrm{KBr}\right): 3173(\mathrm{NH}), 2936(\mathrm{CH}), 1684(\mathrm{CO})$; Anal calcd. for $\mathrm{C}_{18} \mathrm{H}_{16} \mathrm{~N}_{2} \mathrm{O}_{2}$ (CHN).

5-(3,4-Dimethoxybenzyl)-3-phenyl-1,6-dihydro-6-pyridazinone (22): Yield: 56\%; m.p. 198-200 ${ }^{\circ} \mathrm{C}$; ${ }^{1} \mathrm{H}-\mathrm{NMR}\left(\mathrm{CDCl}_{3}, \delta, \mathrm{ppm}\right): 3.63\left(\mathrm{~s}, 2 \mathrm{H}, 2 \mathrm{OOCH}_{3}\right), 3.86\left(\mathrm{~s}, 2 \mathrm{H}, \mathrm{CH}_{2}\right), 7.26(\mathrm{~s}, 1 \mathrm{H}, \mathrm{H}-4$, pyridazinone ring), $7.28(\mathrm{~m}, 1 \mathrm{H}, \mathrm{H}-4$, phenyl ring), $7.38(\mathrm{~m}, 1 \mathrm{H}, \mathrm{H}-6$, benzyl $), 7.61(\mathrm{~m}, 2 \mathrm{H}, \mathrm{H}-$ 3,5 , phenyl ring), $7.64(\mathrm{~m}, 1 \mathrm{H}, \mathrm{H}-5$, benzyl), $7.66(\mathrm{~m}, 1 \mathrm{H}, \mathrm{H}-2$, benzyl $), 11.31(\mathrm{~s}, 1 \mathrm{H}, \mathrm{NH})$; MS $(\mathrm{m} / \mathrm{z}): 322\left(\mathrm{M}^{+}\right) ; \mathrm{IR}\left(\mathrm{cm}^{-1}, \mathrm{KBr}\right): 3167(\mathrm{NH}), 3002(\mathrm{CH}), 1673(\mathrm{CO}) ;$ Anal calcd. for $\mathrm{C}_{19} \mathrm{H}_{18} \mathrm{~N}_{2} \mathrm{O}_{3}$ (CHN).

5-(4-Hydroxy-3-methoxybenzyl)-3-phenyl-1,6-dihydro-6-pyridazinone (23): Yield: 62\%; m.p. $191-193{ }^{\circ} \mathrm{C} ;{ }^{1} \mathrm{H}-\mathrm{NMR}\left(\mathrm{CDCl}_{3}, \delta, \mathrm{ppm}\right): 3.48\left(\mathrm{~s}, 2 \mathrm{H}, \mathrm{CH}_{2}\right), 3.64\left(\mathrm{~s}, 3 \mathrm{H}, \mathrm{OCH}_{3}\right), 6.53(\mathrm{~s}, 1 \mathrm{H}, \mathrm{H}-4$, pyridazinone ring), 7.07-7.29 (m, 5H, phenyl), 7.39-7.72 (3H, H-2,5,6, benzyl), $10.82(\mathrm{~s}, 1 \mathrm{H}$, $\mathrm{NH}) ; \mathrm{MS}(\mathrm{m} / \mathrm{z}): 308\left(\mathrm{M}^{+}\right)$; IR $\left(\mathrm{cm}^{-1}, \mathrm{KBr}\right): 3185(\mathrm{NH}), 2955(\mathrm{CH}), 1686(\mathrm{CO})$; Anal calcd. for $\mathrm{C}_{18} \mathrm{H}_{16} \mathrm{~N}_{2} \mathrm{O}_{3}(\mathrm{CHN})$.

5-(4-Fluorobenzyl)-3-phenyl-1,6-dihydro-6-pyridazinone (24): Yield: 57\%; m.p. $201{ }^{\circ} \mathrm{C}$; ${ }^{1} \mathrm{H}$ $\mathrm{NMR}\left(\mathrm{CDCl}_{3}, \delta, \mathrm{ppm}\right): 3.57\left(\mathrm{~s}, 2 \mathrm{H}, \mathrm{CH}_{2}\right), 7.09(\mathrm{~s}, 1 \mathrm{H}, \mathrm{H}-4$, pyridazinone ring $), 7.28(\mathrm{~m}, 1 \mathrm{H}, \mathrm{H}-$ 4, phenyl), 7.29 (d, H-2,6, p-fluorobenzyl), 7.37 (m, 2H, H-2,6, phenyl), 7.53 (m, $2 \mathrm{H}, \mathrm{H}-3,5, p-$ fluorobenzyl), $7.57\left(\mathrm{~m}, 2 \mathrm{H}, \mathrm{H}-3,5\right.$, phenyl), $11.73(\mathrm{~s}, 1 \mathrm{H}, \mathrm{NH}) ; \operatorname{MS}(\mathrm{m} / z): 280\left(\mathrm{M}^{+}\right) ; \mathrm{R}\left(\mathrm{cm}^{-1}\right.$, $\mathrm{KBr}): 3182(\mathrm{NH}), 2949(\mathrm{CH}), 1673(\mathrm{CO})$; Anal calcd. for $\mathrm{C}_{17} \mathrm{H}_{13} \mathrm{FN}_{2} \mathrm{O}:(\mathrm{CHN})$.

5-(4-Hydroxy-3-ethoxybenzyl)-3-phenyl-1,6-dihydro-6-pyridazinone (25): Yield: 65\%; m.p. 180 ${ }^{\circ} \mathrm{C} ;{ }^{1} \mathrm{H}-\mathrm{NMR}\left(\mathrm{CDCl}_{3}, \delta, \mathrm{ppm}\right): 1.46\left(\mathrm{t}, 3 \mathrm{H}, \mathrm{OCH}_{2} \mathrm{CH}_{3}\right), 3.48\left(\mathrm{~s}, 2 \mathrm{H}, \mathrm{CH}_{2}\right), 4.07(\mathrm{~m}, 2 \mathrm{H}$, $\left.\mathrm{OCH}_{2} \mathrm{CH}_{3}\right), 6.53(\mathrm{~s}, 1 \mathrm{H}, \mathrm{H}-4$, pyridazinone ring), 7.07-7.29 (m, $5 \mathrm{H}$, phenyl), 7.39-7.72 (3H, H2,5,6, benzyl), $10.82(\mathrm{~s}, 1 \mathrm{H}, \mathrm{NH})$; MS $(\mathrm{m} / \mathrm{z}): 322\left(\mathrm{M}^{+}\right)$; IR $\left(\mathrm{cm}^{-1}, \mathrm{KBr}\right): 3184(\mathrm{NH}), 2966(\mathrm{CH})$, 1678 (CO); Anal calcd. for $\mathrm{C}_{19} \mathrm{H}_{18} \mathrm{~N}_{2} \mathrm{O}_{3}(\mathrm{CHN})$. 


\section{Antitubercular Activity of Some Newer 6-Pyridazinone Derivatives}

5-(2-Chlorobenzyl-3-(4-chlorophenyl)-1,6-dihydro-6-pyridazinone (26): Yield: 63\%; m.p. 186$188^{\circ} \mathrm{C} ;{ }^{1} \mathrm{H}-\mathrm{NMR}\left(\mathrm{CDCl}_{3}, \delta, \mathrm{ppm}\right): 3.93\left(\mathrm{~s}, 2 \mathrm{H}, \mathrm{CH}_{2}\right), 7.21(\mathrm{~s}, 1 \mathrm{H}, \mathrm{H}-4$, pyridazinone ring $), 7.28$ (m, 2H, H-4,6, phenyl), 7.38 (m, $1 \mathrm{H}, \mathrm{H}-5$, phenyl ring), 7.40 \& 7.59 (d, each, $2 \times \mathrm{A}_{2} \mathrm{~B}_{2}, p-$ chlorophenyl ring), $7.44(\mathrm{~m}, 1 \mathrm{H}, \mathrm{H}-3$, phenyl), $11.52(\mathrm{~s}, 1 \mathrm{H}, \mathrm{NH}) ; \mathrm{MS}(\mathrm{m} / \mathrm{z}): 330 / 331 / 333$ $\left(\mathrm{M}^{+} / \mathrm{M}+1 / \mathrm{M}+3\right)$; IR $\left(\mathrm{cm}^{-1}, \mathrm{KBr}\right): 3185(\mathrm{NH}), 2952(\mathrm{CH}), 1676(\mathrm{CO}), 714(\mathrm{C}-\mathrm{Cl})$; Anal calcd. for $\mathrm{C}_{17} \mathrm{H}_{12} \mathrm{Cl}_{2} \mathrm{~N}_{2} \mathrm{O}$ (CHN).

5-(2-Hydroxybenzyl)-3-(4-chlorophenyl)-1,6-dihydro-6-pyridazinone (27): Yield: 58\%; m.p. $182-184{ }^{\circ} \mathrm{C} ;{ }^{1} \mathrm{H}-\mathrm{NMR}\left(\mathrm{CDCl}_{3}, \delta, \mathrm{ppm}\right): 3.61\left(\mathrm{~s}, 2 \mathrm{H}, \mathrm{CH}_{2}\right), 7.26(\mathrm{~s}, 1 \mathrm{H}, \mathrm{H}-4$, pyridazinone ring), 7.34 (m, 1H, H-4, phenyl ring), $7.41 \& 7.62$ (d, each, $2 \mathrm{xA}_{2} \mathrm{~B}_{2}, p$-chlorophenyl), $7.64(\mathrm{~m}, 2 \mathrm{H}, \mathrm{H}-$ 2.5, phenyl), 8.37 (m, 1H, H-3, phenyl), $8.57(\mathrm{~s}, 1 \mathrm{H}, \mathrm{OH}), 9.33(\mathrm{~s}, 1 \mathrm{H}, \mathrm{NH}) ; \mathrm{MS}(\mathrm{m} / \mathrm{z}): 312 / 313$ $\left(\mathrm{M}^{+} / \mathrm{M}+1\right)$; IR $\left(\mathrm{cm}^{-1}, \mathrm{KBr}\right): 3174(\mathrm{NH}), 2939(\mathrm{CH}), 1683(\mathrm{CO}), 718(\mathrm{C}-\mathrm{Cl})$; Anal calcd. for $\mathrm{C}_{17} \mathrm{H}_{13} \mathrm{ClN}_{2} \mathrm{O}_{2}(\mathrm{CHN})$.

5-(3-Hydroxybenzyl)-3-(4-chlorophenyl)-1,6-dihydro-6-pyridazinone (28): Yield: 54\%; m.p. 189 ${ }^{\circ} \mathrm{C} ;{ }^{1} \mathrm{H}-\mathrm{NMR}\left(\mathrm{CDCl}_{3}, \delta, \mathrm{ppm}\right): 3.64\left(\mathrm{~s}, 2 \mathrm{H}, \mathrm{CH}_{2}\right), 7.19(\mathrm{~s}, 1 \mathrm{H}, \mathrm{H}-4$, pyridazinone ring $), 7.42 \&$ 7.55 (d, each, $2 \mathrm{xA}_{2} \mathrm{~B}_{2}$, p-chlorophenyl), 7.57 (m, $1 \mathrm{H}, \mathrm{H}-6$, benzyl), 7.98 (m, 1H, H-5, benzyl), $8.07(\mathrm{~m}, 1 \mathrm{H}, \mathrm{H}-4$, benzyl), $8.17(\mathrm{~m}, 1 \mathrm{H}, \mathrm{H}-2$, benzyl), $9.35(\mathrm{~s}, 1 \mathrm{H}, \mathrm{NH}) ; \mathrm{MS}(\mathrm{m} / \mathrm{z}): 312 / 313$ $\left(\mathrm{M}^{+} / \mathrm{M}+1\right)$; IR $\left(\mathrm{cm}^{-1}, \mathrm{KBr}\right): 3168(\mathrm{NH}), 2944(\mathrm{CH}), 1681(\mathrm{CO}), 722(\mathrm{C}-\mathrm{Cl})$; Anal calcd. for $\mathrm{C}_{17} \mathrm{H}_{13} \mathrm{ClN}_{2} \mathrm{O}_{2}$ : (CHN).

5-(3-Nitrobenzyl)-3-(4-chlorophenyl)-1,6-dihydro-6-pyridazinone (29): Yield: 56\%; m.p. $198{ }^{\circ} \mathrm{C}$; ${ }^{1} \mathrm{H}-\mathrm{NMR}\left(\mathrm{CDCl}_{3}, \delta, \mathrm{ppm}\right): 4.05\left(\mathrm{~s}, 2 \mathrm{H}, \mathrm{CH}_{2}\right), 7.15(\mathrm{~s}, 1 \mathrm{H}, \mathrm{H}-4$, pyridazinone ring $), 7.24 \& 7.53$ (d, each, $2 \mathrm{xA}_{2} \mathrm{~B}_{2}, p$-chlorophenyl), 7.55 (m, 1H, H-6, benzyl), 8.21 (m, 1H, H-5, benzyl), 8.31 $(\mathrm{m}, 1 \mathrm{H}, \mathrm{H}-4$, benzyl), $8.49(\mathrm{~m}, 1 \mathrm{H}, \mathrm{H}-2$, benzyl $), 9.23(\mathrm{~s}, 1 \mathrm{H}, \mathrm{NH}) ; \mathrm{MS}(\mathrm{m} / z): 341 / 342$ $\left(\mathrm{M}^{+} / \mathrm{M}+1\right)$; IR $\left(\mathrm{cm}^{-1}, \mathrm{KBr}\right): 3180(\mathrm{NH}), 2934(\mathrm{CH}), 1687(\mathrm{CO}), 717(\mathrm{C}-\mathrm{Cl})$; Anal calcd. for $\mathrm{C}_{17} \mathrm{H}_{12} \mathrm{ClN}_{3} \mathrm{O}_{3}(\mathrm{CHN})$.

5-(3,4-Dimethoxybenzyl)-3-(4-chlorophenyl)-1,6-dihydro-6-pyridazinone (30): Yield: 59\%; m.p. 186-188 ${ }^{\circ} \mathrm{C} ;{ }^{1} \mathrm{H}-\mathrm{NMR}\left(\mathrm{CDCl}_{3}, \delta, \mathrm{ppm}\right): 3.87\left(\mathrm{~s}, 6 \mathrm{H}, 2 \mathrm{x}-\mathrm{OCH}_{3}\right), 3.71\left(\mathrm{~s}, 2 \mathrm{H}, \mathrm{CH}_{2}\right), 6.81(\mathrm{~s}, 1 \mathrm{H}$, $\mathrm{H}-4$, pyridazinone ring), $6.83(\mathrm{~m}, 2 \mathrm{H}, \mathrm{H}-2,6$, benzyl), $7.26(\mathrm{~m}, 1 \mathrm{H}, \mathrm{H}-5$, benzyl), $7.39 \& 7.61(\mathrm{~d}$, each, $2 \mathrm{xA}_{2} \mathrm{~B}_{2}$, p-chlorophenyl), $11.54(\mathrm{~s}, 1 \mathrm{H}, \mathrm{NH}) ; \mathrm{MS}(\mathrm{m} / z): 356 / 357\left(\mathrm{M}^{+} / \mathrm{M}+1\right) ; \mathrm{R}\left(\mathrm{cm}^{-1}\right.$, $\mathrm{KBr}): 3176(\mathrm{NH}), 2959(\mathrm{CH}), 1681(\mathrm{CO}), 725(\mathrm{C}-\mathrm{Cl})$; Anal calcd. for $\mathrm{C}_{19} \mathrm{H}_{17} \mathrm{ClN}_{2} \mathrm{O}_{3}(\mathrm{CHN})$.

\section{Antitubercular activity ${ }^{15,16}$}

The antitubercular screening was carried out against Mycobacterium tuberculosis $\mathrm{H}_{37} \mathrm{Rv}$ (ATCC 27294 ) in Middle brook 7H11 agar medium with OADC (oleic acid albumin dextrose catalase) growth supplement. 10 fold serial dilutions of each test compound/drug (in DMSO/Water mixture) were incorporated into the agar medium. Inoculum of $M$. tuberculosis $\mathrm{H}_{37} \mathrm{Rv}$ were prepared from fresh Middlebrook $7 \mathrm{H} 11$ agar slants with OADC growth supplement adjusted to 1 $\mathrm{mg} / \mathrm{mL}$ (wet weight) in Tween $80(0.05 \%)$ saline diluted to $10^{-2}$ to give a concentration of approximately $10^{7} \mathrm{cfu} / \mathrm{mL}$. A $5 \mu \mathrm{L}$ amount of bacterial suspension was spotted into $7 \mathrm{H} 11$ agar tubes containing 10-fold serial dilutions of drugs per $\mathrm{mL}$. The tubes were incubated at $37^{\circ} \mathrm{C}$, and final readings were recorded after 30 days. The minimum inhibitory concentration (MIC) is defined as the minimum concentration of compound required to give complete inhibition of bacterial growth. The MIC of the standard drug streptomycin was $10 \mu \mathrm{g} / \mathrm{mL}$. The results are presented in Table 1.

\section{RESULTS AND DISCUSSION}

\section{Synthesis}

The starting material, 3-(4-chlorobenzoyl/benzoyl)propionic acid $(1,2)$ was synthesized from dry benzene or chlorobenzene following Friedal Craft's acylation reaction conditions ${ }^{14} .2(3 H)$ Furanones (3-16) were prepared using 3-aroylopionic acid $(1,2)$ following the previously 
SOUTH. BRAZ. J. CHEM., Vol.19, No. 19, 2011

A. Husain, A. Ahmad, A. Bhandari and V. Ram
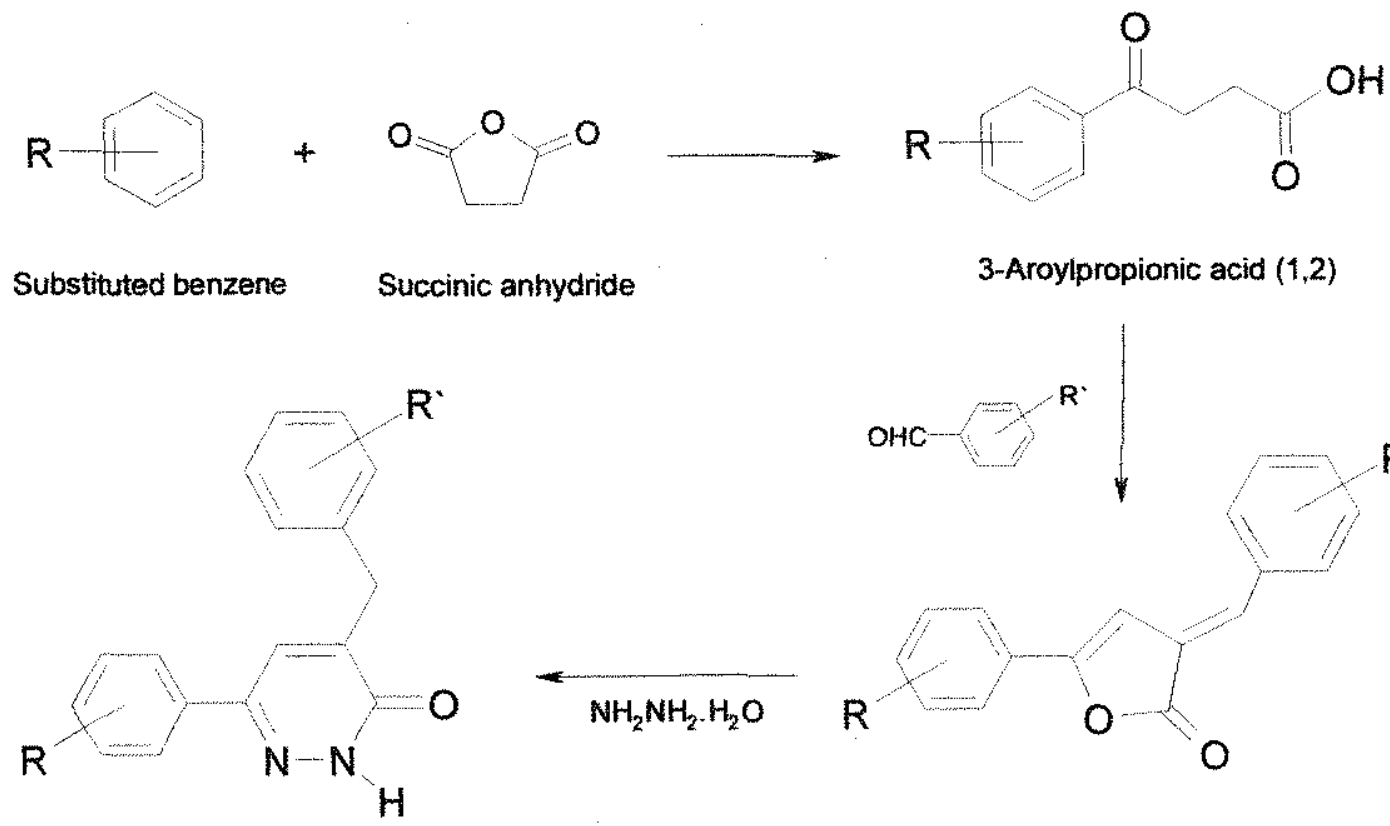

3-Aroylpropionic acid $(1,2)$

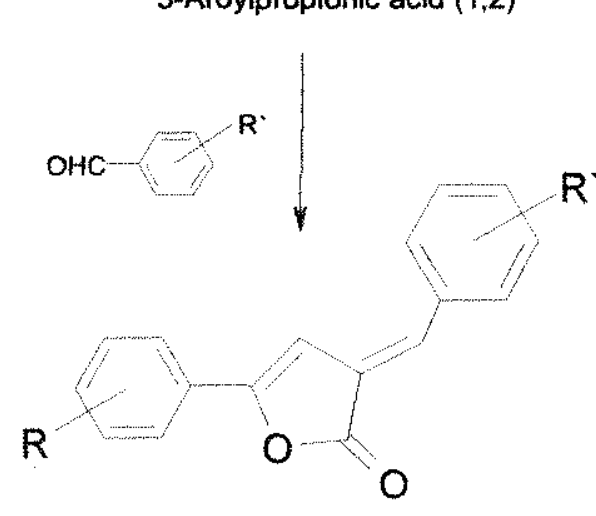

Pyridazinones $(17-30)$

Furanones (3-16)

Scheme 1: Protocol for synthesis of title compounds

Table 1: Antitubercular activity of the 6-pyridazinone derivatives 17-30.

\begin{tabular}{cccc}
\hline Compound & $\mathrm{R}$ & $\mathrm{R}$ & MIC values \\
\hline 17 & $\mathrm{H}$ & $\mathrm{H}$ & 50 \\
18 & $\mathrm{H}$ & $2-\mathrm{Cl}$ & 25 \\
19 & $\mathrm{H}$ & $4-\mathrm{Cl}$ & 50 \\
20 & $\mathrm{H}$ & $3-\mathrm{NO}_{2}$ & 50 \\
21 & $\mathrm{H}$ & $4-\mathrm{OCH}_{3}$ & 50 \\
22 & $\mathrm{H}$ & $3,4-\left(\mathrm{OCH}_{3}\right)_{2}$ & 50 \\
23 & $\mathrm{H}$ & $4-\mathrm{OH} ; 3-\mathrm{OCH}_{3}$ & 12.5 \\
24 & $\mathrm{H}$ & $4-\mathrm{F}$ & 50 \\
25 & $\mathrm{H}$ & $4-\mathrm{OH} ; 3-\mathrm{OC}_{2} \mathrm{H}_{5}$ & 25 \\
26 & $4-\mathrm{Cl}$ & $2-\mathrm{Cl}$ & 50 \\
27 & $4-\mathrm{Cl}$ & $2-\mathrm{OH}$ & 25 \\
28 & $4-\mathrm{Cl}$ & $3-\mathrm{OH}_{2}$ & 50 \\
29 & $4-\mathrm{Cl}$ & $3-\mathrm{NO}_{2}$ & 50 \\
30 & $4-\mathrm{Cl}$ & $3,4-\left(\mathrm{OCH}_{3}\right)_{2}$ & 50 \\
\hline
\end{tabular}




\author{
Antitubercular Activity of Some Newer 6-Pyridazinone Derivatives
}

benzyl)-3-aryl-1,6-dihydro-6-pyridazinone derivatives (17-30) (Scheme-1). Spectral data and microanalysis data were in agreement with the proposed structures.

\title{
Antitubercular activity
}

The antitubercular screening was carried out against Mycobacterium tuberculosis $\mathrm{H}_{37} \mathrm{Rv}$ (ATCC 27294) (Table 1). The results indicated that 5-(4-hydroxy-3-methoxybenzyl)-3-phenyl-1,6dihydro-6-pyridazinone (23) showed best antitubercular activity among the synthesized compounds with $M / C-12.5 \mu \mathrm{g} / \mathrm{mL}$. Three compounds, 5-(2-chlorobenzyl)-3-phenyl-1,6-dihydro6-pyridazinone (18), 5-(4-hydroxy-3-ethoxybenzyl)-3-phenyl-1,6-dihydro-6-pyridazinone (25) and 5-(2-hydroxybenzyl)-3-(4-chlorophenyl)-1,6-dihydro-6-pyridazinone (27) were also significant in their antitubercular action with $M I C-25 \mu \mathrm{g} / \mathrm{mL}$. Rests of the compounds showed $M I C$-values of $50 \mu \mathrm{g} / \mathrm{mL}$. Disubstituted phenyl rings having hydroxyl group $(23 \& 25)$ at $5^{\text {th }}$ position of pyridazinone ring showed good antitubercular activity than unsubstituted or monosubstituted phenyl rings. Among the mono-substituted phenyl rings at $5^{\text {th }}$ position of pyridazinone ring, presence of 2-chloro or 2-hydroxyl group (18 \& 27) showed significant antitubercular activity.

\section{Conclusions}

To sum up, among the synthesized 14 newer pyridazinones, compound 23,5 -(4-hydroxy-3methoxybenzyl)-3-phenyl-1,6-dihydro-6-pyridazinone emerged as lead compound with good antitubercular activity. The study showed the antitubercular potential of 6-pyridazinone derivatives.

Acknowledgements: The authors are thankful to UGC for financial assistance under majorresearch project scheme. We are also thankful to Prof. MSY Khan, Professor Emeritus, Jamia Handard, New Delhi for his valuable suggestions.

\section{REFERENCES}

1. Bloom, B. R.; Murray, C. J. L. Science 1992, 257, 1055.

2. Bames, P. F.; Blotch, A. B.; Davidson, P. T.; Snider, D. E. N. Engl. J. Med. 1991, 324, 1644.

3. Sonmez, M.; Berber, I.; Akbas, E. Eur. J. Med. Chem. 2006, 4l, 101.

4. Abubshait, S.A. Molecules 2007, 12, 25.

5. Rossotti, R.; Rusconi, S. HIV Therapy 2009, 3, 63.

6. Ahmad, S.; Rathish, I. G.; Bano, S.; Alam, M. S.; Javed, K. J. Enzy Inh. Med. Chem. 2010, 25,266 .

7. Dogruer, D. S.; Sahin, M. F.; Unlu, S.; Ito, S. Arch Pharm (Weinheim) 2000, 333, 79.

8. Dogruer, D.; Sahin, M. F. Turk. J. Chem. 2003, 27, 727.

9. Sivakumar, R.; Anbalagan, N.; Gunasekaran, V.; Leonard, J.T. Biol. Pharm. Bull. 2003, 26, 1407.

10. Costas, T., Besada, P., Piras, A., Acevedo, L., Yanez, M., Orallo, F., Laguna, R., Teran, C. Bioorg Med Chem Lett. 2010, 20, 6624.

11. Okushima, A., Narimatsu, A., Kobayashi, M., Furuya, R., Tsuda, K., Kitada, Y. J. Med. Chem. 1987, 30, 1157. 
12. Yamada, T., Nobuhara, Y., Shimamura, H., Yoshihara, K., Yamaguchi, A., Ohki, M. Chem. Pharm. Bull. 1981, 29, 3433.

13. Ali, M. A.; Yar, M. S.; Siddiqui, A. A.; Husain, A.; Abdullah, M. Acta Polo. Pharm-Drug Research 2007, 63, 435.

14. Husain, A.; Alam, M. M.; Hasan, S. M.; Yar, M. S. Acta Polo. Pharm-Drug Research $2009,66,173$.

15. Elmer, W. K.; Stephen, D. A.; William, M. J.; Paul, C. S.; Washing Jr C. W. 'Text book of diagnostic Microbiology' $5^{\text {th }}$ edn, Lippincot Publishers, 2002.

16. Ellen, J. B.; Lancer, R. P.; Sydney, M. F. 'Bailey and Scott's Diagnostic Microbiology' $9^{\text {th }}$ edn, 2000.

VISIT OUR SITE: http://www.sbjchem.he.com.br 